\title{
CONVERGENCE ESTIMATES FOR MULTIGRID ALGORITHMS WITHOUT REGULARITY ASSUMPTIONS
}

\author{
JAMES H. BRAMBLE, JOSEPH E. PASCIAK, JUNPING WANG, AND JINCHAO XU
}

\begin{abstract}
A new technique for proving rate of convergence estimates of multigrid algorithms for symmetric positive definite problems will be given in this paper. The standard multigrid theory requires a "regularity and approximation" assumption. In contrast, the new theory requires only an easily verified approximation assumption. This leads to convergence results for multigrid refinement applications, problems with irregular coefficients, and problems whose coefficients have large jumps. In addition, the new theory shows why it suffices to smooth only in the regions where new nodes are being added in multigrid refinement applications.
\end{abstract}

\section{INTRODUCTION}

In recent years, multigrid methods have been used extensively as tools for obtaining approximations to solutions of partial differential equations (see the references in [13], [14], [20]). In conjunction, there has been intensive research into the theoretical understanding of the convergence properties of the methods (cf. [3]-[10], [14], [17], [19]). This paper will present some new results on the convergence of multigrid algorithms. In particular, we shall prove multigrid convergence results under regularity-free assumptions.

The standard multigrid analysis uses a "regularity and approximation" assumption [6], [20]. This hypothesis is proved using elliptic regularity for the solution of the underlying partial differential equation as well as approximation and inverse properties of the discrete multilevel spaces. In contrast, two-level schemes have been proved to converge under apparently weaker assumptions [12], [14], [16], [18], [22], [23]. These arguments often only require approximation properties of the subspace. In this paper, we shall prove convergence

Received April 17, 1990; revised July 24, 1990.

1980 Mathematics Subject Classification (1985 Revision). Primary 65N30; Secondary 65F10.

This manuscript has been authored under contract number DE-AC02-76CH00016 with the U.S. Department of Energy. Accordingly, the U.S. Government retains a nonexclusive, royalty-free license to publish or reproduce the published form of this contribution, or allow others to do so, for U.S. Government purposes. This work was also supported in part under the National Science Foundation Grant No. DMS84-05352, DMS88-05311-04 and by the U.S. Army Research Office through the Mathematical Sciences Institute, Cornell University. The third author's research was supported by Office of Naval Research Contract No. 0014-88-K-0370 and by the Institute for Scientific Computation of the University of Wyoming through National Science Foundation Grant No. RII-8610680. 
estimates for general multilevel procedures under these weaker approximation assumptions. Somewhat weaker results of this form are proved in [7].

In the case of mesh refinement, the regularity and approximation constants may grow with some power of the ratio of the finest to coarsest mesh size. Our theory will show that the rate of convergence for these examples is no worse than $1-c / j$, where $j$ is the number of levels in the multigrid scheme. In addition, our analysis explains why it is only necessary to smooth in the region where refined triangles (or nodes) are being added.

Other applications of the theory to be provided include general $H_{0}^{1}$ equivalent forms and operators whose coefficients have large jumps. Both of the above-mentioned multigrid applications satisfy the approximation assumption, and hence the theory of this paper provides a fundamental analysis. In contrast, an analysis proving regularity and approximation estimates for these cases is either unavailable or leads to inequalities with large constants.

We should note that there has been some attempt to generalize the two-level analysis to the multilevel case [1], [21]. For example, [21] provides an analysis for an algorithm which requires applying a Chebyshev iterative scheme for the coarse grid correction, the number of terms depending on the convergence rate of the two-level scheme. The work estimates for this scheme are the same as those for the general multigrid algorithm with the number of correction steps ( $p$ in Algorithm $\mathrm{S}$ or $\mathrm{N}$ ) equal to the number of terms in the Chebyshev series and hence is unacceptable for large $p$. The analysis presented here provides an estimate for the multilevel algorithm (including the V-cycle) without placing any restrictions on the convergence rate of the two-level algorithm. This is important since, as far as we know, there is no computationally effective way of improving the theoretical convergence rate of the two-level algorithm without assuming the regularity and approximation property.

The outline of the remainder of the paper is as follows. In $\S 2$, following [6], we define the multigrid algorithms and derive some fundamental recurrence relations. In $\S 3$, we give the regularity-free multigrid convergence analysis. In $\S 4$, we consider the application of the earlier theory to the case of general $H_{0}^{1}$ equivalent forms. Finite element and finite difference applications are considered there. An example in which the grid is locally refined and smoothing is only on the refined subregion is given in $\S 5$. In $\S 6$, we consider the applications to problems which have large jumps in coefficients. In $\S 7$, we give the results of numerically computed convergence factors which illustrate the theory developed in this paper. Finally, we include an appendix in which some issues concerning the implementation of the schemes are considered.

\section{THE MULTIGRID ALGORITHMS}

In this section, following [6], we describe the multigrid algorithms in both the symmetric and nonsymmetric cases. Along the way, we derive basic recurrence relations which play major roles in the analysis of the methods. For convenience, the algorithms are developed in an abstract Hilbert space setting. The 
results most naturally apply to finite element multigrid algorithms but can also be applied to certain formulations of finite difference multigrid algorithms.

Let us assume that we are given a nested sequence of finite-dimensional vector spaces

$$
M_{0} \subset M_{1} \subset \cdots \subset M_{j} .
$$

In addition, let $A(\cdot, \cdot)$ and $(\cdot, \cdot)_{k}$ be symmetric positive definite bilinear forms on $M_{k}$ for $k=0, \ldots, j$. We shall develop multigrid algorithms for the solution of the problem: Given $f \in M_{j}$, find $v \in M_{j}$ satisfying

$$
A(v, \phi)=(f, \phi)_{j} \text { for all } \phi \in M_{j} .
$$

To define the multigrid algorithms, we shall define auxiliary operators. For $k=0, \ldots, j$, define the operator $A_{k}: M_{k} \mapsto M_{k}$ by

$$
\left(A_{k} w, \phi\right)_{k}=A(w, \phi) \quad \text { for all } \phi \in M_{k} \text {. }
$$

The operator $A_{k}$ is clearly symmetric (in both the $A(\cdot, \cdot)$ - and $(\cdot, \cdot)_{k}$-inner products) and positive definite. Also define the projectors $P_{k}: M_{j} \mapsto M_{k}$ and operators $P_{k}^{0}: M_{k+1} \mapsto M_{k}$ by

$$
A\left(P_{k} w, \phi\right)=A(w, \phi) \quad \text { for all } \phi \in M_{k},
$$

and

$$
\left(P_{k}^{0} w, \phi\right)_{k}=(w, \phi)_{k+1} \quad \text { for all } \phi \in M_{k} .
$$

Note that $P_{k}$ is symmetric in the $A$-inner product.

To define the smoothing process, we require a linear operator $R_{k}: M_{k} \mapsto M_{k}$ for $k=1, \ldots, j$. We assume that $R_{k}$ is a symmetric and positive semidefinite operator in the $(\cdot, \cdot)_{k}$-inner product and set $K_{k}=I-R_{k} A_{k}$ on $M_{k}$. Clearly, $K_{k}$ is symmetric in the inner product $A(\cdot, \cdot)$, and we further assume that $K_{k}$ is nonnegative in the sense that $A\left(K_{k} u, u\right) \geq 0$ for all $u \in M_{k}$. These assumptions imply that the spectrum of $K_{k}$ is in $[0,1]$.

We first define the symmetric multigrid operator $B_{k}^{s}: M_{k} \mapsto M_{k}$ by induction.

Algorithm S. Set $B_{0}^{s}=A_{0}^{-1}$. Assume that $B_{k-1}^{s}$ has been defined and define $B_{k}^{s} g$ for $g \in M_{k}$ as follows:

(1) Set $x^{0}=0$ and $q^{0}=0$.

(2) Define $x^{l}$ for $l=1, \ldots, m(k)$ by

$$
x^{l}=x^{l-1}+R_{k}\left(g-A_{k} x^{l-1}\right) .
$$

(3) Define $x^{m(k)+1}=x^{m(k)}+q^{p}$ where $q^{i}$ for $i=1, \ldots, p$ is defined by

$$
q^{i}=q^{i-1}+B_{k-1}^{s}\left[P_{k-1}^{0}\left(g-A_{k} x^{m(k)}\right)-A_{k-1} q^{i-1}\right] .
$$

(4) Set $B_{k}^{s} g=x^{2 m(k)+1}$ where $x^{l}$ is defined for $l=m(k)+2, \ldots, 2 m(k)+1$ by $(2.3)$. 
In this algorithm, $m(k)$ is a positive integer which may vary from level to level and determines the number of smoothing iterations on that level. Because of this variable smoothing, the above algorithm is more general than that usually described [3], [4], [13], [14]. If all of the $m(k)$ are the same, then this algorithm is the usual symmetric multigrid algorithm described in a notation which is convenient for our analysis. Note that $B_{k}^{s}$ is clearly a linear operator for each $k$. In this algorithm, $p$ is a positive integer. The cases $p=1$ and $p=2$ correspond respectively to the symmetric $\mathscr{V}$ and $\mathscr{W}$ cycles of multigrid.

The definition of the nonsymmetric multigrid operator $B_{k}^{n}$ is similar except that the smoothings of Step 4 are excluded. More precisely, we define $B_{k}^{n}: M_{k} \mapsto$ $M_{k}$ by induction.

Algorithm N. Set $B_{0}^{n}=A_{0}^{-1}$. Assume that $B_{k-1}^{n}$ has been defined and define $B_{k}^{n} g$ for $g \in M_{k}$ as follows:

(1) Set $x^{0}=0$ and $q^{0}=0$.

(2) Define $x^{l}$ for $l=1, \ldots, m(k)$ by (2.3).

(3) Define $B_{k}^{n} g=x^{m(k)}+q^{p}$ where $q^{i}$ for $i=1, \ldots, p$ is defined by

$$
q^{i}=q^{i-1}+B_{k-1}^{n}\left[P_{k-1}^{0}\left(g-A_{k} x^{m(k)}\right)-A_{k-1} q^{i-1}\right] .
$$

The above algorithm defines a linear operator $B_{k}^{n}$ which is equivalent to the standard nonsymmetric multigrid algorithms described in [4], [14] when $m(k)$ is constant.

Let $g=A_{k} x$. It is straightforward to check that $q^{p}$ defined by (2.4) satisfies

$$
q^{p}=\left(I-\left(I-B_{k-1}^{n} A_{k-1}\right)^{p}\right) A_{k-1}^{-1} P_{k-1}^{0} A_{k}\left(x-x^{m(k)}\right) .
$$

A trivial computation gives that

$$
x-x^{m(k)}=K_{k}^{m(k)} x .
$$

Noting that on $M_{k}, P_{k-1}^{0} A_{k}=A_{k-1} P_{k-1}$, and combining (2.5) and (2.6) gives

$$
I-B_{k}^{n} A_{k}=\left[\left(I-P_{k-1}\right)+\left(I-B_{k-1}^{n} A_{k-1}\right)^{p} P_{k-1}\right] K_{k}^{m(k)} \text { on } M_{k} \text {. }
$$

Equation (2.7) gives a fundamental recurrence relation for the nonsymmetric multigrid algorithms. The analogous recurrence in the symmetric case is

$$
I-B_{k}^{s} A_{k}=K_{k}^{m(k)}\left[\left(I-P_{k-1}\right)+\left(I-B_{k-1}^{s} A_{k-1}\right)^{p} P_{k-1}\right] K_{k}^{m(k)} \text { on } M_{k},
$$

which follows from similar reasoning.

We now restrict our attention to the case of $p=1$. Note that $K_{k}=K_{k}^{*}$, where $K_{k}^{*}$ is the adjoint with respect to the inner product $A(\cdot, \cdot)$. Consequently,

$$
\left(I-B_{k}^{n} A_{k}\right)^{*}=K_{k}^{m(k)}\left[\left(I-P_{k-1}\right)+\left(I-B_{k-1}^{n} A_{k-1}\right)^{*} P_{k-1}\right] .
$$

Multiplying this with (2.7), we find by a straightforward induction argument

$$
I-B_{j}^{s} A_{j}=\left(I-B_{j}^{n} A_{j}\right)^{*}\left(I-B_{j}^{n} A_{j}\right) \text {. }
$$


Let $\|\mid \cdot\|$ denote the norm corresponding to the inner product $A(\cdot, \cdot)$ on $M_{j}$. Clearly, the convergence rate of Algorithm $\mathrm{S}$ is bounded by the operator norm of $I-B_{j}^{s} A_{j}$ with respect to \|\|$\cdot \| \mid$, which is the square of the operator norm of $I-B_{j}^{n} A_{j}$.

For our analysis, we will write $I-B_{j}^{n} A_{j}$ as a product of operators. By (2.7), for $k=1, \ldots, j$, we clearly have that, on $M_{j}$,

$$
\begin{aligned}
I-B_{k}^{n} A_{k} P_{k} & =\left(I-P_{k}\right)+\left(I-B_{k-1}^{n} A_{k-1} P_{k-1}\right) K_{k}^{m(k)} P_{k} \\
& =\left(I-B_{k-1}^{n} A_{k-1} P_{k-1}\right)\left(I-P_{k}+K_{k}^{m(k)} P_{k}\right) \\
& =\left(I-B_{k-1}^{n} A_{k-1} P_{k-1}\right)\left(I-T_{k}\right),
\end{aligned}
$$

where $T_{k}=\left(I-K_{k}^{m(k)}\right) P_{k}$. Note that we have used the fact that $P_{k-1}\left(I-P_{k}\right)=0$ in the derivation of the above equality. For convenience, we define $T_{0}=P_{0}$ and hence

$$
I-B_{j}^{n} A_{j}=\left(I-T_{0}\right)\left(I-T_{1}\right) \cdots\left(I-T_{j}\right),
$$

from which it follows that

$$
\left(I-B_{j}^{n} A_{j}\right)^{*}=\left(I-T_{j}\right) \cdots\left(I-T_{1}\right)\left(I-T_{0}\right),
$$

since $T_{k}^{*}=T_{k}$. We note that each factor of (2.11) is defined on all of $M_{j}$. To develop bounds for the convergence of either Algorithm S or N, it suffices to estimate the operator norm of $\left(I-B_{j}^{n} A_{j}\right)^{*}$.

The more general case $p \geq 1$ is quite similar. We first consider the nonsymmetric algorithm. Set

$$
\varepsilon_{k}=\left(I-B_{k}^{n} A_{k} P_{k}\right)^{*} \text { for } k=0, \ldots, j .
$$

A manipulation similar to that used above yields $\varepsilon_{k}=\left(I-T_{k}\right) \varepsilon_{k-1}^{p}$ for $k=$ $1, \ldots, j$. Thus,

$$
\begin{aligned}
\varepsilon_{j} & =\left(I-T_{j}\right)\left(I-T_{j-1}\right) \varepsilon_{j-2}^{p} \varepsilon_{j-1}^{p-1} \\
& =\left(I-T_{j}\right) \cdots\left(I-T_{1}\right)\left(I-T_{0}\right) \varepsilon_{1}^{p-1} \varepsilon_{2}^{p-1} \cdots \varepsilon_{j-1}^{p-1}
\end{aligned}
$$

Clearly, $\left\|\mid \varepsilon_{1}^{p-1} \varepsilon_{2}^{p-1} \cdots \varepsilon_{j-1}^{p-1}\right\| \| \leq 1$. Consequently, estimates for Algorithm $\mathrm{N}$ with $p>1$ follow from the estimates for Algorithm $\mathrm{N}$ with $p=1$. Similar arguments applied to the symmetric algorithm imply that

$$
\begin{aligned}
I-B_{j}^{s} A_{j}= & \left(I-T_{j}\right) \cdots\left(I-T_{1}\right)\left(I-T_{0}\right) D_{j} \\
& \cdot\left(I-T_{0}\right)\left(I-T_{1}\right) \cdots\left(I-T_{j}\right),
\end{aligned}
$$

where the $\|\cdot \cdot\| \mid$ norm of the operator $D_{j}$ is also bounded by one. Consequently, the convergence rate for $p>1$ is always bounded by the convergence rate for the case of $p=1$. Our analysis will not guarantee an improved rate of convergence for $p>1$ under the regularity-free assumption (3.3). In contrast, the proofs 
assuming the regularity and approximation assumption [6], [14], [20] do suggest such improvements.

\section{Multigrid ANALYSIS}

In this section, we give an analysis of the multigrid algorithms described in the previous section. Our goal is to prove inequalities of the form:

$$
A\left(\left(I-B_{j}^{s} A_{j}\right) u, u\right) \leq \delta_{j} A(u, u) \text { for all } u \in M_{j},
$$

or

$$
A\left(\left(I-B_{j}^{n} A_{j}\right) u,\left(I-B_{j}^{n} A_{j}\right) u\right) \leq \delta_{j} A(u, u) \text { for all } u \in M_{j} \text {. }
$$

From the previous discussion, it suffices to prove (3.2) when $p=1$.

In contrast to the usual multigrid analysis [6], [20], we will relate $\delta_{j}$ in (3.2) to somewhat different a priori assumptions. Let $\lambda_{k}$ denote the largest eigenvalue of $A_{k}$. Our main assumption is the existence of linear operators $Q_{k}: M_{j} \mapsto M_{k}$ for $k=0,1, \ldots, j$, with $Q_{j}=I$, satisfying the following properties. We assume that there are constants $C_{1}$ and $C_{2}$ not depending on $k$ for which

$$
\begin{aligned}
\left\|\left(Q_{k}-Q_{k-1}\right) u\right\|_{k}^{2} & \leq C_{1} \lambda_{k}^{-1} A(u, u) \text { for } k=1, \ldots, j, \\
A\left(Q_{k} u, Q_{k} u\right) & \leq C_{2} A(u, u) \text { for } k=0, \ldots, j-1 .
\end{aligned}
$$

The inequalities in (3.3) hold for all $u \in M_{j}$. As will be demonstrated in $\S \S 4-6$, in various applications the derivation of inequalities of the form (3.3) only uses the approximation properties of the subspaces.

We will also make a second assumption concerning the symmetric smoothing operator $R_{k}$. Assume that $\widetilde{M}_{k} \equiv$ "the range of $R_{k}$ " contains the range of $Q_{k}-Q_{k-1}$. We further assume that there exists a constant $C_{R}$ independent of $k$ which satisfies

$$
\frac{\|u\|_{k}^{2}}{\lambda_{k}} \leq C_{R}\left(R_{k} u, u\right)_{k} \quad \text { for all } u \in \widetilde{M}_{k}
$$

Let $\widetilde{P}_{k}^{0}$ denote the orthogonal projection of $M_{k}$ onto $\widetilde{M}_{k}$ with respect to $(\cdot, \cdot)_{k}$. We note that for $v \in M_{k}$ and $\phi \in \widetilde{M}_{k}$,

$$
\left(R_{k} \tilde{P}_{k}^{0} v, \phi\right)_{k}=\left(v, R_{k} \phi\right)_{k}=\left(R_{k} v, \phi\right)_{k}
$$

i.e., $R_{k} \widetilde{P}_{k}^{0}=R_{k}$.

Remark 3.1. The above assumption on $R_{k}$ represents a minimal requirement for a symmetric operator to be considered a smoother and is a natural condition to be satisfied. The fact that $R_{k}$ need not be definite on $M_{k}$ plays an important role in certain applications. In effect, the above assumption only requires that $R_{k}$ smooth on the range of $Q_{k}-Q_{k-1}$. In a refinement application described in $\S 5, \widetilde{M}_{k}$ will only contain degrees of freedom in a refinement subdomain and hence smoothing need only be done in that subdomain. Algorithms which only 
smooth in the refinement regions have been suggested in [2]. An example of an $R_{k}$ satisfying (3.4) is $R_{k}=\bar{\lambda}_{k}^{-1} \widetilde{P}_{k}^{0}$, where $\bar{\lambda}_{k}$ is any upper bound for $\lambda_{k}$ (we need $K_{k}$ nonnegative) and $\widetilde{P}_{k}^{0}$ is the orthogonal projection of $M_{k}$ onto $\widetilde{M}_{k}$ with respect to $(\cdot, \cdot)_{k}$.

We can no'w state and prove the theorem for estimating $\delta_{j}$ in (3.1) and (3.2).

Theorem 1. Assume that (3.3) and (3.4) hold, and define $B_{j}^{s}$ and $B_{j}^{n}$ by Algorithm $\mathrm{S}$ and $\mathrm{N}$, respectively. Then (3.1) and (3.2) hold with

$$
\delta_{j}=1-\frac{1}{C j},
$$

where $C=\left[1+C_{2}^{1 / 2}+\left(C_{R} C_{1}\right)^{1 / 2}\right]^{2}$.

Proof. From the earlier discussion, we may assume, without loss of generality, that $p=1$. In this case, (3.1) and (3.2) are equivalent. Let $E_{k}=\left(I-B_{k}^{n} A_{k} P_{k}\right)^{*}$. By (2.10), we have for $k=1, \ldots, j$

$$
E_{k}=\left(I-T_{k}\right) E_{k-1} \text {. }
$$

Equality (3.6) also holds for $k=0$ provided that we define $E_{-1}=I$.

We note that to prove (3.2) for $\delta_{j}$ given by (3.5), it suffices to show that

$$
A(u, u) \leq C j\left[A(u, u)-A\left(E_{j} u, E_{j} u\right)\right] .
$$

We start from (3.6) and compute

$$
\begin{aligned}
A\left(E_{k-1} u, E_{k-1} u\right)= & A\left(E_{k} u, E_{k} u\right)+2 A\left(T_{k}\left(I-T_{k}\right) E_{k-1} u, E_{k-1} u\right) \\
& +A\left(T_{k} E_{k-1} u, T_{k} E_{k-1} u\right) \\
= & A\left(E_{k} u, E_{k} u\right)+A\left(T_{k}\left(I-T_{k}\right) E_{k-1} u, E_{k-1} u\right) \\
& +A\left(T_{k} E_{k-1} u, E_{k-1} u\right) .
\end{aligned}
$$

From the definition of $T_{k}$, the operator $T_{k}\left(I-T_{k}\right)$ is symmetric with respect to $A(\cdot, \cdot)$ and positive semidefinite, and hence

$$
A\left(T_{k} E_{k-1} u, E_{k-1} u\right) \leq A\left(E_{k-1} u, E_{k-1} u\right)-A\left(E_{k} u, E_{k} u\right) .
$$

Summing from 0 to $j$ gives

$$
\sum_{k=0}^{j} A\left(T_{k} E_{k-1} u, E_{k-1} u\right) \leq A(u, u)-A\left(E_{j} u, E_{j} u\right) .
$$

By (3.7) and (3.9), (3.5) is reduced to proving that

$$
A(u, u) \leq C j \sum_{k=0}^{j} A\left(T_{k} E_{k-1} u, E_{k-1} u\right) .
$$

To this end, we use the fact that $Q_{j}=I$ to write

$$
u=\sum_{k=1}^{j}\left(Q_{k}-Q_{k-1}\right) u+Q_{0} u
$$


Thus,

$$
\begin{aligned}
& A(u, u)= \sum_{k=1}^{j} A\left(u,\left(Q_{k}-Q_{k-1}\right) u\right)+A\left(u, Q_{0} u\right) \\
&=\sum_{k=1}^{j} A\left(E_{k-1} u,\left(Q_{k}-Q_{k-1}\right) u\right)+A\left(u, Q_{0} u\right) \\
& \quad+\sum_{k=1}^{j} A\left(\left(I-E_{k-1}\right) u,\left(Q_{k}-Q_{k-1}\right) u\right) .
\end{aligned}
$$

We now estimate the first sum on the right of (3.11). Using Schwarz's inequality, we see that

$$
\begin{aligned}
\sum_{k=1}^{j} A\left(E_{k-1} u,\left(Q_{k}-Q_{k-1}\right) u\right) & =\sum_{k=1}^{j} A\left(P_{k} E_{k-1} u,\left(Q_{k}-Q_{k-1}\right) u\right) \\
& =\sum_{k=1}^{j}\left(\widetilde{P}_{k}^{0} A_{k} P_{k} E_{k-1} u,\left(Q_{k}-Q_{k-1}\right) u\right)_{k} \\
& \leq \sum_{k=1}^{j}\left\|\widetilde{P}_{k}^{0} A_{k} P_{k} E_{k-1} u\right\|_{k}\left\|\left(Q_{k}-\dot{Q}_{k-1}\right) u\right\|_{k} .
\end{aligned}
$$

Applying (3.3) and (3.4) gives

$$
\begin{aligned}
& \sum_{k=1}^{j} A\left(E_{k-1} u,\left(Q_{k}-Q_{k-1}\right) u\right) \\
& \quad \leq\left(C_{1}\right)^{1 / 2} A^{1 / 2}(u, u) \sum_{k=1}^{j} \lambda_{k}^{-1 / 2}\left\|\widetilde{P}_{k}^{0} A_{k} P_{k} E_{k-1} u\right\|_{k} \\
& \quad \leq\left(C_{R} C_{1} j\right)^{1 / 2} A^{1 / 2}(u, u)\left(\sum_{k=1}^{j} A\left(R_{k} A_{k} P_{k} E_{k-1} u, P_{k} E_{k-1} u\right)\right)^{1 / 2}
\end{aligned}
$$

We used the fact that $R_{k} \widetilde{P}_{k}^{0}=R_{k}$ in the last inequality above. The spectrum of $K_{k}$ is in $[0,1]$, and hence

$$
\begin{aligned}
A\left(R_{k} A_{k} P_{k} E_{k-1} u, P_{k} E_{k-1} u\right) & =A\left(\left(I-K_{k}\right) P_{k} E_{k-1} u, P_{k} E_{k-1} u\right) \\
& \leq A\left(T_{k} E_{k-1} u, E_{k-1} u\right) .
\end{aligned}
$$

Thus, we obtain

$$
\begin{aligned}
& \sum_{k=1}^{j} A\left(E_{k-1} u,\left(Q_{k}-Q_{k-1}\right) u\right) \\
& \quad \leq\left(C_{1} C_{R} j\right)^{1 / 2} A^{1 / 2}(u, u)\left(\sum_{k=1}^{j} A\left(T_{k} E_{k-1} u, E_{k-1} u\right)\right)^{1 / 2} .
\end{aligned}
$$


For the remaining terms in (3.11), we have

$$
\begin{aligned}
& \sum_{k=1}^{j} A\left(\left(I-E_{k-1}\right) u,\left(Q_{k}-Q_{k-1}\right) u\right)+A\left(u, Q_{0} u\right) \\
& \quad=\sum_{k=1}^{j-1} A\left(\left(E_{k}-E_{k-1}\right) u, Q_{k} u\right)+A\left(\left(I-E_{j-1}\right) u, u\right)
\end{aligned}
$$

From (3.6), $E_{k-1}-E_{k}=T_{k} E_{k-1}$, and it follows that

$$
I-E_{j-1}=\sum_{k=0}^{j-1} T_{k} E_{k-1} .
$$

From this and (3.14),

$$
\begin{array}{r}
\sum_{k=1}^{j} A\left(\left(I-E_{k-1}\right) u,\left(Q_{k}-Q_{k-1}\right) u\right)+A\left(u, Q_{0} u\right) \\
\quad=\sum_{k=1}^{j-1} A\left(T_{k} E_{k-1} u,\left(I-Q_{k}\right) u\right)+A\left(T_{0} u, u\right) .
\end{array}
$$

Note that the spectrum of $T_{k}$ is contained in $[0,1]$, and hence the right-hand side of (3.15) is bounded by

$$
j^{1 / 2}\left(1+C_{2}^{1 / 2}\right)\left(\sum_{k=0}^{j-1} A\left(T_{k} E_{k-1} u, E_{k-1} u\right)\right)^{1 / 2} A^{1 / 2}(u, u)
$$

Combining (3.11), (3.13), (3.15), and (3.16) gives

$$
A(u, u) \leq j\left[1+C_{2}^{1 / 2}+\left(C_{R} C_{1}\right)^{1 / 2}\right]^{2}\left(\sum_{k=0}^{j} A\left(T_{k} E_{k-1} u, E_{k-1} u\right)\right)
$$

i.e., (3.10) holds with $C=\left[1+C_{2}^{1 / 2}+\left(C_{R} C_{1}\right)^{1 / 2}\right]^{2}$. This completes the proof of the theorem.

Remark 3.2. Theorem 1 gives bounds for the convergence factor $\delta_{k}$ in (3.1) and (3.2) in terms of $j$. In finite element applications, $j$ (the number of levels) is proportional to a logarithm of the mesh size. This theorem shows that the multigrid algorithms converge rapidly under the regularity-free assumption (3.3). We note, however, that the result does not show any improvement in the rate of convergence when the number of smoothings $m(k)$ is greater than one. In contrast, the multigrid results of [5], [6] imply improved convergence with larger $m(k)$ provided that the regularity and approximation assumption holds.

Remark 3.3. The nonnegativity assumption on $K_{k}$ can be relaxed to the assumption that $\sigma\left(K_{k}\right) \subseteq[1-\omega, 1]$ for some $\omega$ with $1 \leq \omega<2$. Here, $\sigma\left(K_{k}\right)$ denotes the spectrum of $K_{k}$. The value of $C$ in Theorem 1 can be taken to 
be $C=\left[1+C_{2}^{1 / 2}+\left(C_{R} C_{1} \omega /(2-\omega)\right)^{1 / 2}\right]^{2} /(2-\omega)$. The assumption on $\sigma\left(K_{k}\right)$ implies that $\sigma\left(T_{k}\right) \subseteq[0, \omega]$. In this case, (3.8) is replaced by

$$
(2-\omega) A\left(T_{k} E_{k-1} u, E_{k-1} u\right) \leq A\left(E_{k-1} u, E_{k-1} u\right)-A\left(E_{k} u, E_{k} u\right)
$$

and (3.12) is replaced by

$$
A\left(R_{k} A_{k} P_{k} E_{k-1} u, P_{k} E_{k-1} u\right) \leq \frac{\omega}{2-\omega} A\left(T_{k} E_{k-1} u, E_{k-1} u\right) .
$$

The remainder of the proof is similar.

\section{Application to an $H_{0}^{1}$ eQuivalent form}

In this section, we consider applying the multilevel results of the previous section to forms $A(\cdot, \cdot)$ defined on a subspace $M_{j} \subseteq H_{0}^{1}(\Omega)$. We will only assume that the quadratic form is equivalent to the square of the $H^{1}(\Omega)$ norm on $M_{j}$, i.e.,

$$
c_{0}\|v\|_{1}^{2} \leq A(v, v) \leq c_{1}\|v\|_{1}^{2} \text { for all } v \in M_{j}
$$

holds for positive constants $c_{0}, c_{1}$. Here, $\|\cdot\|_{1}^{2}$ denotes the norm in $H^{1}(\Omega)$. As far as we know, it is not possible to prove the regularity and approximation assumption ((3.3) of [6]) under such a weak assumption on the form. In contrast, we shall see that it is possible to apply the theory of the preceding section to natural multigrid algorithms and guarantee rapid convergence. Examples for which (4.1) is known, yet regularity and approximation results are not available, include elliptic problems with bounded measurable coefficients and various finite difference applications.

We start with a nested sequence of multigrid spaces

$$
M_{0} \subset M_{1} \subset \cdots \subset M_{j} .
$$

The most natural examples result from finite element subspaces defined on a sequence of nested triangulations. For example, in the two-dimensional case, when $\Omega$ is a polygonal domain, we can define spaces of piecewise linear functions as follows. Since $\Omega$ is polygonal, start with a coarse triangulation $\tau_{0}=$ $\bigcup_{l} \tau_{0}^{l}$ of quasi-uniform size $h_{0}$, where $\tau_{0}^{l}$ represents an individual triangle and $\tau_{0}$ denotes the triangulation. Successively finer triangulations $\left\{\tau_{k}, k=\right.$ $1, \ldots, j\}$ of size $h_{k}=2^{-k} h_{0}$ are defined by breaking each triangle of a coarser triangulation into four triangles by connecting the midpoints of the edges. The subspace $M_{k}$ is defined to be the continuous functions defined on $\Omega$ which are piecewise linear with respect to $\tau_{k}$ and vanish on $\partial \Omega$.

Let $Q_{k}$ denote the $L^{2}(\Omega)$ projection onto $M_{k}$. It is known that, since the triangulations are quasi-uniform,

$$
\left\|\left(I-Q_{k}\right) v\right\| \leq c h_{k}\|v\|_{1}
$$

and

$$
\left\|Q_{k} v\right\|_{1} \leq C\|v\|_{1},
$$


for all $v \in H_{0}^{1}(\Omega)$ (see, e.g., [11], [23]). Here, $\|\cdot\|$ denotes the norm in $L^{2}(\Omega)$. Let $(\cdot, \cdot)_{k}$ be a discrete inner product on $M_{k} \times M_{k}$ which satisfies

$$
c\|v\|^{2} \leq(v, v)_{k} \leq C\|v\|^{2} \text { for all } v \in M_{k} \text {. }
$$

The constants $c$ and $C$ in (4.4) are assumed to be independent of $k=$ $0, \ldots, j$. For example, we can take

$$
(u, v)_{k}=h_{k}^{2} \sum u\left(x_{i}\right) v\left(x_{i}\right),
$$

where $\left\{x_{i}\right\}$ is the collection of nodes for $M_{k}$ and the sum in (4.5) is taken over this set. Combining (4.1)-(4.4) shows that (3.3) holds. Since, in this case, we use discrete inner products, we can take $R_{k}$ to be $\bar{\lambda}_{k}^{-1} I$, where $\bar{\lambda}_{k}=O\left(h_{k}^{-2}\right)$ is a bound for the largest eigenvalue of $A_{k}$. Consequently, we can apply Theorem 1 to provide the following proposition.

Proposition 4.1. Assume that (4.1)-(4.4) hold. Then the convergence rates of Algorithms $\mathrm{S}$ and $\mathrm{N}$ are bounded by

$$
\delta_{j}=1-\frac{1}{C j} .
$$

As the first application of this result, we will consider the following elliptic boundary value problem. Let $\Omega$ be a polygonal domain in $R^{2}$ and consider the problem

$$
\begin{aligned}
-\sum_{i, j=1}^{2} \frac{\partial}{\partial x_{i}} a_{i j} \frac{\partial u}{\partial x_{j}}+a u & =f \quad \text { in } \Omega, \\
u & =0 \quad \text { on } \partial \Omega .
\end{aligned}
$$

We assume that the matrix $\left\{a_{i j}\right\}$ is symmetric for each $x \in \Omega$. The corresponding form $A(\cdot, \cdot)$ is defined by

$$
A(u, v)=\sum_{i, j=1}^{2} \int_{\Omega} a_{i j} \frac{\partial u}{\partial x_{j}} \frac{\partial v}{\partial x_{i}} d x+\int_{\Omega} a u v d x .
$$

For this application, the only additional requirement is that the coefficients are such that (4.1) holds. This means that the coefficients can have rather complex jumps provided that they remain between fixed upper and lower bounds. Proposition 4.1 applies and gives a convergence bound for the corresponding multigrid algorithms. As far as we know, appropriate elliptic regularity estimates (cf. [4], [6]) for the weak solutions of problem (4.6) are not known. Without such estimates, neither the theory of [4] nor [6] would yield useful convergence bounds.

For our second application of Proposition 4.1, we consider a finite difference operator applied to the problem

$$
\begin{array}{rlrl}
-\nabla \cdot a \nabla u=f & \text { in } \Omega, \\
u=0 & & \text { on } \partial \Omega .
\end{array}
$$


For this application, we assume that the domain is the union of squares in a coarse square mesh of size $h_{0}$ and that the coefficient $a$ in (4.8) is continuous on $\bar{\Omega}$ and bounded from below by a positive constant $c$. The coarse finite difference mesh is formed by the above-mentioned squares. Successively finer grids are defined by breaking each square into four subsquares in the obvious way. We seek the solution of the finite difference equations on the $j$ th grid.

The finite difference stencil at the grid point $i, k$ of the $j$ th grid is given by

$$
\begin{aligned}
\left(L_{h} \bar{x}\right)_{i, k}= & a_{i, k+1 / 2}\left(\bar{x}_{i, k}-\bar{x}_{i, k+1}\right)+a_{i, k-1 / 2}\left(\bar{x}_{i, k}-\bar{x}_{i, k-1}\right) \\
& +a_{i+1 / 2, k}\left(\bar{x}_{i, k}-\bar{x}_{i+1, k}\right)+a_{i-1 / 2, k}\left(\bar{x}_{i, k}-\bar{x}_{i-1, k}\right) .
\end{aligned}
$$

We also consider finite element spaces of piecewise linear functions as defined above. The coarse triangulation of $\Omega$ is formed by dividing each of the coarse squares mentioned above into two triangles by one of the diagonals. Nodal interpolation $\bar{x} \rightarrow x$ provides a natural correspondence between the nodal vectors in the finite difference approximation and functions in $M_{j}$, i.e., $x$ is the function in $M_{j}$ which takes on the values of $\bar{x}$ on the nodes of the grid. It is easy to show that the norm induced by the form

$$
A(x, y)=\sum\left(L_{h} \bar{x}\right)_{i, k} \bar{y}_{i, k}
$$

is equivalent to the $H^{1}$ norm on $M_{j}$. The sum in (4.9) is over all nodes of the $j$ th mesh. Consequently, Proposition 4.1 guarantees rates of convergence for the corresponding multigrid algorithms (with discrete inner products given by, for example, (4.5)).

This algorithm corresponds to a natural finite difference multigrid algorithm. In fact, the finite element spaces can be used to provide interpolation and restriction operators connecting the original sequence of finite difference spaces $\bar{M}_{0}, \ldots, \bar{M}_{j}$. Define the $k$ th grid operator $L_{h}^{k}$ by ([20])

$$
L_{h}^{k}=I_{k}^{t} L_{h} I_{k},
$$

where $I_{k}$ is the interpolation matrix (corresponding to the imbedding of $M_{k}$ in $M_{j}$ ) and $I_{k}^{t}$ is its transpose. Then the multigrid algorithm using the form of (4.9) is exactly the same as the finite difference multigrid algorithms using (4.10). Proposition 4.1 provides an estimate for its rate of convergence.

The discussion given in this section extends without difficulty to problems in more than two dimensions and other boundary conditions as well as more general finite element spaces.

\section{A REFINEMENT APPLICATION}

In this section, we shall apply the results of $\S 3$ to the case of finite element approximation with locally refined grids. Such mesh refinements are convenient for accurate modeling of problems with various types of singular behavior. Here, we shall make use of the fact that the operator $R_{k}$ need not satisfy 
smoothing conditions on the whole space but rather only on a subspace containing the range of $Q_{k}-Q_{k-1}$. This will allow an analysis of algorithms which only smooth on the nodes which are interior to the subdomain of refinement.

We consider the boundary value problem (4.6), but for convenience, we take $a=0$. As in $\S 4$, we assume that the coefficient matrix is symmetric and that the resulting form (4.7) satisfies (4.1). It is well known that jumps in coefficients, nonconvex domains, or singular data results in solutions with various types of singular behavior [15]. To accurately solve such problems, it is convenient to use an appropriate mesh refinement. We shall present a flexible mesh refinement scheme in this section which leads to multigrid algorithms whose convergence rate can be bounded by applying Theorem 1 .

For convenience, we will only consider the case of piecewise linear finite elements. Applications with other finite element spaces are possible. Also the case of more than two dimensions presents no inherent difficulty.

The first step in the definition of the algorithms involves the development of sequences of nested refined grids. These grids are defined in terms of a given sequence of nested subdomains

$$
\Omega_{j} \subseteq \Omega_{j-1} \subseteq \cdots \subseteq \Omega_{0}=\Omega .
$$

We assume that we are given a coarse triangulation of $\Omega=\bigcup_{l} \tau_{0}^{l}$. This coarse triangulation provides the first grid $\left\{\tau_{0}^{l}\right\}$. Given that a grid $\left\{\tau_{k-1}^{l}\right\}$ has been defined, the grid $\left\{\tau_{k}^{l}\right\}$ is defined by refining those triangles of $\left\{\tau_{k-1}^{l}\right\}$ which are in $\Omega_{k}$. This refinement is done by breaking each triangle of the mesh $\left\{\tau_{k-1}^{l}\right\}$ in $\Omega_{k}$ into four triangles by connecting the midpoints of the edges. We assume $\partial \Omega_{k}$ aligns with the mesh $\left\{\tau_{k-1}^{l}\right\}$.

The space $M_{k}$ is defined to be the set of continuous functions on $\Omega$ which are piecewise linear with respect to the grid $\left\{\tau_{k}^{l}\right\}$ and vanish on $\partial \Omega$. We note that the continuity constraint implies that there are no new degrees of freedom corresponding to nodes on $\partial \Omega_{k}$ (see Figure 5.1). These new nodes on $\partial \Omega_{k}$ will be called slave nodes since, by continuity, their values are determined by the values of their neighboring nodes (which were already in the previous grids). It is easy to see that the space $M_{k}$ has a nodal basis consisting of the vertices of $\left\{\tau_{k}\right\}$ excluding the slave nodes.

To fit this application into the abstract framework of $\S 2$ and $\S 3$, we need to define discrete inner products and smoothing operators $R_{k}$. We shall use the $L^{2}(\Omega)$-inner product for the discrete inner product on each level. The use of $L^{2}(\Omega)$-inner products in the multigrid algorithms often leads to algorithms which require the solution of Gram matrix problems in the implementation. We shall avoid this (see the Appendix) by a judicious choice of the smoothing operator $R_{k}$. Let

$$
\widetilde{M}_{k}=\left\{\phi \in M_{k} \mid \operatorname{supp} \phi \subseteq \Omega_{k}\right\} .
$$




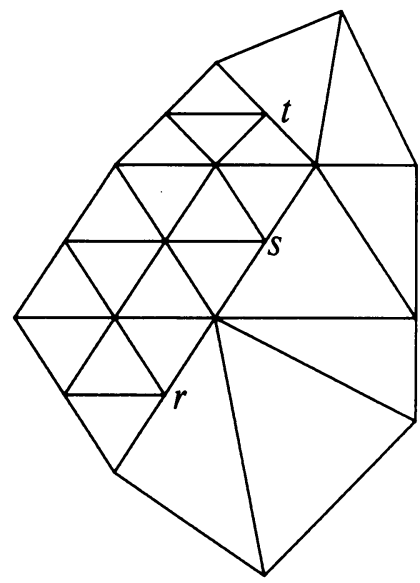

$r, s, t:$ Slave Nodes

FIGURE 5.1

$A$ mesh transition region

In addition, let $(\cdot, \cdot)$ denote the $L^{2}(\Omega)$-inner product and $\left\{\theta_{k}^{l}\right\}$ denote the usual nodal basis for $\widetilde{M}_{k}$. Define

$$
R_{k} u=\alpha \sum_{l}\left(d_{k}^{l}\right)^{-1}\left(u, \theta_{k}^{l}\right) \theta_{k}^{l} \quad \text { for all } u \in M_{k} \text {. }
$$

The sum in (5.2) is taken over the nodes of $\widetilde{M}_{k}, d_{k}^{i}=A\left(\theta_{k}^{i}, \theta_{k}^{i}\right)$ and $\alpha$ is a constant which is chosen to ensure that $K_{k}$ is nonnegative.

In general, it suffices to take $\alpha=1 / 3$. The nonnegativity of $K_{k}$ can be seen as follows. For $u \in M_{k}$,

$$
A\left(R_{k} A_{k} u, u\right)=1 / 3 \sum_{i} A\left(u, \theta_{k}^{i}\right)^{2} A\left(\theta_{k}^{i}, \theta_{k}^{i}\right)^{-1} \leq 1 / 3 \sum_{i} A_{\Omega_{k}^{i}}(u, u)
$$

Here $\Omega_{k}^{i}$ denotes the support of $\theta_{k}^{i}$. Each triangle of the mesh $\left\{\tau_{k}^{l}\right\}$ appears at most three times on the right-hand sum in (5.3). The nonnegativity of $K_{k}$ immediately follows.

The operator $R_{k}$ is clearly a symmetric semidefinite operator on $M_{k}$ with range contained in $\widetilde{M}_{k}$. We note that the space $\widetilde{M}_{k}$ is a finite element space developed from a mesh with elements of quasi-uniform size. It is shown in [9] that (3.4) holds with the discrete norms defined to be the $L^{2}(\Omega)$ norm. Note that this definition of $R_{k}$ provides a smoothing operator which only involves smoothing on the nodes of $M_{k}$ in the interior of the refinement subregion $\Omega_{k}$. We will prove the following proposition.

Proposition 5.1. There exists a sequence of operators $Q_{k}: M_{j} \mapsto M_{k}$ for $k=$ $0, \ldots, j$, with $Q_{j}=I$, satisfying (3.3). Moreover, $\left(Q_{k}-Q_{k-1}\right) v \in \widetilde{M}_{k}$ for all 
$v \in M_{j}$. Consequently, Algorithms $\mathrm{S}$ and $\mathrm{N}$ using $A(\cdot, \cdot), L^{2}(\Omega)$-inner products, and $R_{k}$ by (5.2), converge with rates bounded by

$$
\delta_{j}=1-\frac{1}{C j} .
$$

Proof. We need only construct a sequence of operators $Q_{k}, k=0, \ldots, j-1$, satisfying (3.3) with $\left(Q_{k}-Q_{k-1}\right) v \in \widetilde{M}_{k}$ for all $v \in M_{k}$. To do this, we use a technique given in [9]. Let $h_{0}$ denote the mesh size of the original coarse mesh and define $h_{k}=2^{-k} h_{0}$. We clearly have that $\lambda_{k} \leq c h_{k}^{-2}$. Let $\bar{M}_{k}$ be the space defined from the full mesh of size $h_{k}$, i.e., $\bar{M}_{k}$ is the space obtained above when taking $\Omega_{k}=\Omega_{k-1}=\cdots=\Omega_{0}=\Omega$. Clearly, $\bar{M}_{k}$ satisfies the usual approximation and inverse properties, which imply that the $L^{2}(\Omega)$ projection operator $\bar{Q}_{k}$ onto $\bar{M}_{k}$ satisfies

$$
\left\|\left(I-\bar{Q}_{k}\right) v\right\| \leq c \lambda_{k}^{-1 / 2} A^{1 / 2}(v, v)
$$

and

$$
A\left(\bar{Q}_{k} v, \bar{Q}_{k} v\right) \leq C A(v, v),
$$

for all $v \in H_{0}^{1}(\Omega)$. Moreover, both $M_{k}$ and $\bar{M}_{k}$ have the same mesh restricted to $\Omega_{k}$ and $M_{k} \subseteq \bar{M}_{k}$. Let $v \in M$. For $k=0, \ldots, j-1$, define $Q_{k} v=w$, where $w$ is the unique function in $M_{k}$ satisfying

$$
w= \begin{cases}\bar{Q}_{k} v & \text { at the nodes of } M_{k} \text { in the interior of } \Omega_{k+1}, \\ v & \text { at the remaining nodes of } M_{k} .\end{cases}
$$

Note that from the construction, $\left(Q_{k}-Q_{k-1}\right) v$ is a function in $M_{k}$ with support in $\Omega_{k}$, i.e., it is in $\widetilde{M}_{k}$. We are left to verify (3.3).

We first prove that

$$
\left\|\left(I-Q_{k}\right) v\right\|^{2} \leq c \lambda_{k}^{-1} A(v, v),
$$

from which the first inequality of (3.3) follows. By the definition of $Q_{k}$ and the triangle inequality,

$$
\begin{aligned}
\left\|\left(I-Q_{k}\right) v\right\| & =\|v-w\|=\|v-w\|_{\Omega_{k+1}} \\
& \leq\left\|\left(I-\bar{Q}_{k}\right) v\right\|+\left\|\bar{Q}_{k} v-w\right\|_{\Omega_{k+1}},
\end{aligned}
$$

where $\|\cdot\|_{\Omega_{k+1}}$ denotes the $L^{2}$ norm on $\Omega_{k+1}$. By (5.4), it suffices to estimate the second term on the right-hand side of (5.7) by the first. But by the definition of $w$,

$$
\left\|\bar{Q}_{k} v-w\right\|_{\Omega_{k+1}}^{2} \leq C h_{k}^{2} \sum_{i}\left(\bar{Q}_{k} v\left(x_{k}^{i}\right)-v\left(x_{k}^{i}\right)\right)^{2},
$$

where the sum is taken over the nodes $x_{k}^{i}$ of $M_{k}$ on $\partial \Omega_{k+1}$. Clearly,

$$
h_{k}^{2} \sum_{i}\left(\bar{Q}_{k} v\left(x_{k}^{i}\right)-v\left(x_{k}^{i}\right)\right)^{2} \leq C\left\|\left(I-\bar{Q}_{k}\right) v\right\| \text {. }
$$

This proves (5.6) and completes the proof of the first inequality of (3.3). 
To prove the second inequality of (3.3), it obviously suffices to prove

$$
A\left(\left(I-Q_{k}\right) v,\left(I-Q_{k}\right) v\right) \leq c A(v, v) .
$$

As above, we write

$$
\begin{aligned}
& A^{1 / 2}\left(\left(I-Q_{k}\right) v,\left(I-Q_{k}\right) v\right)=A_{\Omega_{k+1}}^{1 / 2}(v-w, v-w) \\
& \quad \leq A^{1 / 2}\left(\left(I-\bar{Q}_{k}\right) v,\left(I-\bar{Q}_{k}\right) v\right)+A_{\Omega_{k+1}}^{1 / 2}\left(\bar{Q}_{k} v-w, \bar{Q}_{k} v-w\right)
\end{aligned}
$$

Here, $A_{\Omega_{k+1}}(\cdot, \cdot)$ is defined as in (4.7) but with integration taken only over the subdomain $\Omega_{k+1}$. We apply (5.5) to get

$$
A\left(\left(I-\bar{Q}_{k}\right) v,\left(I-\bar{Q}_{k}\right) v\right) \leq C A(v, v) .
$$

For the remaining term in (5.9), as above

$$
\begin{aligned}
A_{\Omega_{k+1}}\left(\bar{Q}_{k} v-w, \bar{Q}_{k} v-w\right) & \leq C \sum_{i}\left(\bar{Q}_{k} v\left(x_{k}^{i}\right)-v\left(x_{k}^{i}\right)\right)^{2} \\
& \leq C h_{k}^{-2}\left\|\left(I-\bar{Q}_{k}\right) v\right\| \leq C A(v, v) .
\end{aligned}
$$

This proves the second inequality of (3.3) and hence completes the proof of the proposition.

Remark 5.1. The use of $L^{2}(\Omega)$-inner products for discrete inner products sometimes leads to multigrid algorithms which require the solution of Gram matrix problems. However, the special form of the operators $R_{k}$ enables us to avoid such complications. This is discussed in detail in the appendix.

\section{INTERFACE PROBLEMS WITH LARGE JUMPS IN COEFFICIENTS}

As the final example of the applications of the theory in $\S 3$, in this section we will present some multigrid estimates for second-order problems which may have large jumps in coefficients. We consider the following problem defined on a domain $\Omega \subset R^{2}$ :

$$
\begin{aligned}
-\nabla(a \nabla) u=f & \text { in } \Omega, \\
u=0 & \text { on } \partial \Omega .
\end{aligned}
$$

More precisely, we assume

$$
\bar{\Omega}=\bigcup_{i=1}^{J} \bar{\Omega}_{i},
$$

where $\left\{\Omega_{i}\right\}$ are mutually disjoint open polygons or tetrahedrons, and set $\omega_{i}$ to be the average value of $a$ over $\Omega_{i}$. In particular, we shall be interested in applications when there is large variation in $\left\{\omega_{i}\right\}$ but little variation of $a$ over the sets $\Omega_{i}$. The coefficient $a$ may not be smooth, e.g., it might have jump discontinuities, in which case (6.1) is understood in the weak sense. Consequently, 
we assume that $\omega_{i}>0$ for each $i$, and that there are constants $c_{0}$ and $c_{1}$ not depending on $i=1, \ldots, J$ satisfying

$$
c_{0} \omega_{i} D_{i}(v, v) \leq A_{i}(v, v) \leq c_{1} \omega_{i} D_{i}(v, v) \text { for all } v \in H^{1}\left(\Omega_{i}\right) .
$$

Here, $A_{i}(\cdot, \cdot)$ is defined by

$$
A_{i}(u, v)=\int_{\Omega_{i}} a \nabla u \cdot \nabla v d x .
$$

Similarly, $D_{i}(\cdot, \cdot)$ denotes the Dirichlet form on $\Omega_{i}$ and

$$
A(u, v)=\sum_{i} A_{i}(u, v)
$$

The purpose of this section is to apply the results of $\S 3$ to provide estimates for appropriately defined multigrid algorithms for (6.1) which depend only on the constants $c_{0}, c_{1}$ of $(6.3)$ but not on the values of $\omega_{i}$. This means that $\max _{i} \omega_{i} / \min _{i} \omega_{i}$ can be very large without significantly reducing the rate of convergence for these multigrid algorithms. To achieve this rate of convergence, we use a discrete inner product which is weighted by the coefficients $\left\{\omega_{i}\right\}$ and a coarse triangulation which aligns with the boundaries of $\left\{\boldsymbol{\Omega}_{i}\right\}$. By this we mean that $\bigcup_{i} \partial \Omega_{i}$ is a subset of $\bigcup_{l} \partial \tau_{0}^{l}$, where $\left\{\tau_{0}^{l}\right\}$ is the coarse triangulation of $\Omega$ (see $\S 4)$.

As far as we know, the dependence of the elliptic regularity estimates (in the weighted norms) is not known for this type of problem. Consequently, the regularity and approximation assumptions necessary for the standard theory are not available. Some analysis for a fixed number of levels has been given in [23] although the estimates given there tend rapidly to one as the number of levels is increased. In contrast, the bounds we shall derive here only deteriorate quadratically with the number of levels (see Proposition 6.1).

To begin our analysis, we introduce the following weighted inner products:

$$
(u, v)_{L_{\omega}^{2}(\Omega)}=\sum_{i=1}^{J} \omega_{i}(u, v)_{L^{2}\left(\Omega_{i}\right)},
$$

and

$$
(u, v)_{H_{\omega}^{1}(\Omega)}=\sum_{i=1}^{J} \omega_{i} D_{i}(u, v),
$$

with the induced norms denoted by $\|\cdot\|_{L_{\omega}^{2}(\Omega)}$ and $\|\cdot\|_{H_{\omega}^{1}(\Omega)}$, respectively. Notice that by $(6.3), A^{1 / 2}(\cdot, \cdot)$ is equivalent to $\|\cdot\|_{H_{\omega}^{1}(\Omega)}$. As is done in $\S 4$, we assume that $\Omega$ is triangulated by a nested sequence of quasi-uniform meshes $\left\{\tau_{k}: k=0, \ldots, j\right\}$ with $\left\{\partial \Omega_{i}\right\}$ being a subgrid of $\left\{\tau_{0}^{l}\right\}$. Corresponding to these triangulations, as in $\S 4$, we have the multilevel spaces

$$
M_{0} \subset M_{1} \subset \cdots \subset M_{j}
$$


The operators $Q_{k}$ needed in the analysis of $\S 3$ can be taken to be the weighted $L^{2}$ projections $Q_{k}^{\omega}: L^{2}(\Omega) \mapsto M_{k}$ defined by

$$
\left(Q_{k}^{\omega} u, v\right)_{L_{\omega}^{2}(\Omega)}=(u, v)_{L_{\omega}^{2}(\Omega)} \text { for all } u \in L^{2}(\Omega), v \in M_{k} .
$$

The following result is taken from [23] for the verification of (3.3).

Lemma 6.1. Assume the decomposition (6.2) has no cross points, namely there is no point on the interface that belongs to more than two $\bar{\Omega}_{i}$ 's. Then, for all $u \in H_{0}^{1}(\Omega)$,

$$
\left\|\left(I-Q_{k}^{\omega}\right) u\right\|_{L_{\omega}^{2}(\Omega)} \leq C h_{k}\|u\|_{H_{\omega}^{1}(\Omega)}
$$

and

$$
\left\|Q_{k}^{\omega} u\right\|_{H_{\omega}^{1}(\Omega)} \leq C\|u\|_{H_{\omega}^{1}(\Omega)} .
$$

More generally, in the presence of cross points, we have, for all $u \in M_{j}$,

$$
\left\|\left(I-Q_{k}^{\omega}\right) u\right\|_{L_{\omega}^{2}(\Omega)} \leq C h_{k}\left(\log \frac{h_{k}}{h_{j}}\right)^{1 / 2}\|u\|_{H_{\omega}^{1}(\Omega)}
$$

and

$$
\left\|Q_{k}^{\omega} u\right\|_{H_{\omega}^{1}(\Omega)} \leq C\left(\log \frac{h_{k}}{h_{j}}\right)^{1 / 2}\|u\|_{H_{\omega}^{1}(\Omega)} .
$$

Remark 6.1. The first part of the above result holds in three dimensions but, in general, the second does not [23].

To completely define the multigrid algorithms, we need only define the discrete inner products and the smoothing operators $\left\{R_{k}\right\}$. The discrete inner products are defined by the weighted inner product $(6.5)$. We define the operator $R_{k}$ by

$$
R_{k} v=\alpha \sum_{i}\left(d_{k}^{i}\right)^{-1}\left(v, \theta_{k}^{i}\right)_{L_{\omega}^{2}(\Omega)} \theta_{k}^{i},
$$

Here, $d_{k}^{i}=A\left(\theta_{k}^{i}, \theta_{k}^{i}\right)$ and $\alpha$ is a constant chosen so that $K_{k}$ is nonnegative. From the discussion in $\S 5$, it suffices to take $\alpha=1 / 3$.

By the inverse inequality, we have that the largest eigenvalue of $A_{k}$ (defined by (2.2)) is bounded by $C h_{k}^{-2}$. As a consequence, Lemma 6.1 implies that the assumption (3.3) holds with $C_{1}$ and $C_{2}$ satisfying

$$
C_{1} \leq \widehat{C}_{1} j^{\gamma}, \quad C_{2} \leq \widehat{C}_{2} j^{\gamma},
$$

where $\hat{C}_{1}$ and $\hat{C}_{2}$ are constants independent of $k$ and $j$. The constant $\gamma$ is equal to zero if the interface has no cross points and otherwise $\gamma$ equals one. Applying Theorem 1 gives the following proposition. 
Proposition 6.1. For the operators and inner products defined above, the convergence rate of Algorithm $\mathrm{S}$ and $\mathrm{N}$ are bounded by

$$
\delta_{j} \leq 1-\frac{1}{C j^{1+\gamma}},
$$

where $\gamma=0$ or 1 as explained above.

Remark 6.2. The above proposition holds for three-dimensional applications provided that, for example, there are no internal cross points.

Remark 6.3. The special form of the smoothing operators $R_{k}$ defined in (6.8) enables us to avoid the solution of Gram matrix problems associated with the inner products appearing in the multigrid algorithms. This is discussed in detail in the appendix.

\section{NUMERICAL RESULTS}

In this section, we provide the results of numerical examples illustrating the theory developed in the earlier sections. Specifically, the actual reduction factor $\delta_{j}$ satisfying (3.1) is numerically computed in several examples. Note that $\delta_{j}$ is the largest eigenvalue of the operator $I-B_{j} A_{j}$ and can be computed numerically. We shall provide results in the case of local refinement (see $\$ 5$ ) as well as quasi-uniform meshes applied to problems where the coefficients defining the differential operator have jumps (see $\S \S 4$ and 6). The results of the refinement calculations appear to be independent of the number of levels. In contrast, the examples in which the coefficients have jumps show a slow deterioration in the rate of convergence as the number of levels increases. Both cases show asymptotic convergence behavior which is somewhat better than the worst-case analysis provided by the earlier theory. In all of the reported results, we use a symmetric V-cycle algorithm with $m(k)=1$ for all $k$.

We report the numerically computed value of $\delta_{j}$ as a function of the mesh parameters. We note however that, since the operator $B_{j}$ is symmetric, it can be used as a preconditioner and the overall convergence of the algorithm can be accelerated by preconditioned conjugate gradient iteration. In this case, the condition number of the preconditioned system is bounded by $\left(1-\delta_{j}\right)^{-1}$.

For the first example, we consider the application of the multigrid algorithm to the finite element equations corresponding to a problem with mesh refinement. For this example, the domain $\Omega$ will be the unit square, and we shall approximate the solution to

$$
\begin{aligned}
-\Delta u=f & \text { in } \Omega, \\
u=0 & \text { on } \partial \Omega .
\end{aligned}
$$

The corresponding form $A(\cdot, \cdot)$ is the Dirichlet form on $\Omega$.

The sequence of grids which we shall consider will be progressively more refined as we approach the corner $(1,1)$. We will use the scheme described in $\S 5$ for generating the mesh. We start by breaking the square into sixteen smaller 
squares of side length $1 / 4$. The coarse triangulation is defined by splitting each of these smaller squares into two triangles, for example, along the diagonal between the bottom left to the upper right corner. For integers $0<J<j$, we define $\Omega_{k}=\Omega$ for $k=0, \ldots, J$ and $\Omega_{k}=\left[1-2^{J-k}, 1\right] \times\left[1-2^{J-k}, 1\right]$ for $k>J$. This generates a sequence of meshes with geometrically decreasing mesh size, with local refinement (for $k>J$ ) on domains of geometrically decreasing size. Such a mesh would be effective if, for example, the function $f$ in (7.1) behaved like a $\delta$ function distribution at the point $(1,1)$. The finite element spaces $M_{0} \subset \cdots \subset M_{j}$ are defined as in $\S 5$.

We use (5.2) to define $R_{k}$. A more careful analysis shows that, for this example, $I-R_{k} A_{k}$ will be a nonnegative operator if $\alpha$ is taken to be $1 / 2$. We define $(\cdot, \cdot)_{k}$ to be the $L^{2}(\Omega)$-inner product.

Table 7.1 gives numerically computed values of $\delta_{j}$ as a function of $j$ and $J$. For example, $J=4$ and $j=8$ corresponds to a uniform grid of size $h_{J}=1 / 64$ with four refinement levels in the corner and $h_{j}=1 / 1024$. For all practical purposes, these values are independent of both $j$ and $J$. In contrast, Proposition 5.1 suggests that they may deteriorate like $1-c / j$. In this example, all of the domains $\Omega_{0}, \ldots, \Omega_{j}$ are squares and the worst-case deterioration does not appear to be occurring.

TABLE 7.1

Values of $\delta_{j}$ for the refinement example

\begin{tabular}{|c|c|c|c|c|}
\hline$j-J$ & $J=1$ & $J=2$ & $J=3$ & $J=4$ \\
\hline 1 & .670 & .668 & .668 & .668 \\
2 & .669 & .668 & .668 & .668 \\
3 & .669 & .668 & .668 & .668 \\
4 & .669 & .668 & .668 & .668 \\
\hline
\end{tabular}

The remaining example considers multigrid applied to a problem with jumps in the coefficients. Specifically, we consider finite element approximation of the solution of the problem (6.1) where $\Omega$ is the unit square and the coefficient $a(x)$ is piecewise constant on the coarse grid triangles. Actually, $a(x)=1$ except in the set $[1 / 4,1 / 2] \times[1 / 4,1 / 2] \cup[1 / 2,3 / 4] \times[1 / 2,3 / 4]$, where it is equal to $\mu$. We shall present numerically computed values of $\delta_{j}$ for $\mu=$ $1,2,1000,10000$.

For this example, we use quasi-uniform grids and subspaces corresponding to $k=0, \ldots, J$ described in the previous example. The form $A(\cdot, \cdot)$ is given by (6.4) and $R_{k}$ is defined by (6.8). As in the previous example, we take $\alpha=1 / 2$.

Table 7.2 gives the numerically computed values of $\delta_{j}$ as a function of $j$ and $\mu$. Note that the results for $\mu=1,2$ appear to be bounded independently of the number of levels. For $\mu=2$, this is somewhat better than the bound of Proposition 4.1. In addition, little change is seen in the reduction rates 
of $\mu=1,000$ and $\mu=10,000$. Both $\mu=1,000$ and $\mu=10,000$ show a deterioration in the rate of convergence which is better than the theoretical bound of $1-c / j^{2}$ provided by Proposition 6.1 ; in fact, $\delta_{j}$ is approximately $1-.8 / j$ (see the last column of Table 7.2).

TABLE 7.2

Values of $\delta_{j}$ for the discontinuous coefficient example

\begin{tabular}{|c|c|c|c|c|c|}
\hline$j\left(h_{j}\right)$ & $\mu=1$ & $\mu=2$ & $\mu=1,000$ & $\mu=10,000$ & $1-.8 / j$ \\
\hline $2(1 / 16)$ & .57 & .59 & .62 & .62 & .60 \\
$3(1 / 32)$ & .59 & .61 & .72 & .73 & .73 \\
$4(1 / 64)$ & .59 & .61 & .80 & .80 & .80 \\
$5(1 / 128)$ & .59 & .61 & .84 & .85 & .84 \\
\hline
\end{tabular}

\section{APPENDIX}

In this section, we shall consider the implementation of Algorithms $\mathrm{S}$ and $\mathrm{N}$. In particular, we shall show that it is sometimes possible to implement these algorithms in a way which is independent of the discrete inner products. This is possible for the applications described in $\S 5$ and $\S 6$.

Before proceeding, we briefly examine the way finite element equations are described in computer codes. Typically, one represents unknown functions in an approximation space (e.g., $M_{k}$ ) by a vector of nodal coefficients. The computer problem is to find the coefficients of the function $u \in M_{k}$ satisfying

$$
A\left(u, \theta_{k}^{i}\right)=F\left(\theta_{k}^{i}\right),
$$

for all basis function $\left\{\theta_{k}^{i}\right\}$. The functional $F$ is represented by its action on the basis vectors. One defines the stiffness matrix $M$ by $\tilde{A}_{i j}=A\left(\theta_{k}^{i}, \theta_{k}^{j}\right)$. Note that, in terms of these representations, the stiffness matrix corresponds to a linear operator $\tilde{A}_{k}$ from $M_{k}$ onto $M_{k}^{\prime}$ (the space of linear functionals on $M_{k}$ ).

As we shall see, it is possible to implement a multigrid algorithm with inner products which are defined from a fixed inner product $(\cdot, \cdot)$ provided that the smoothing operator $R_{k}$ has the form

$$
R_{k} u=\sum_{i} r_{k}^{i}\left(u, \theta_{k}^{i}\right) \theta_{k}^{i} .
$$

Here, $\left\{r_{k}^{i}\right\}$ is a vector of coefficients and $(\cdot, \cdot)$ is a fixed inner product independent of $k$. We will consider the case of Algorithm S; the case of Algorithm $\mathrm{N}$ is similar.

Corresponding to $R_{k}$, we define the linear operator $\widetilde{R}_{k}: M_{k}^{\prime} \mapsto M_{k}$ by

$$
\widetilde{R}_{k} F=\sum_{i} r_{k}^{i} F\left(\theta_{k}^{i}\right) \theta_{k}^{i} \quad \text { for all } F \in M_{k}^{\prime} .
$$


Instead of defining a sequence of operators $B_{k}: M_{k} \mapsto M_{k}, k=0, \ldots, j$, we define, by induction, a sequence of operators $\widetilde{B}_{k}: M_{k}^{\prime} \mapsto M_{k}, k=0, \ldots, j$, as follows:

Set $\widetilde{B}_{0}^{s}=\widetilde{A}_{0}^{-1}$. Assume that $\widetilde{B}_{k-1}^{s}$ has been defined and define $\widetilde{B}_{k}^{s} g$ for $g \in M_{k}^{\prime}$ as follows:

(1) Set $x^{0}=0$ and $q^{0}=0$.

(2) Define $x^{l}$ for $l=1, \ldots, m(k)$ by

$$
x^{l}=x^{l-1}+\widetilde{R}_{k}\left(g-\widetilde{A}_{k} x^{l-1}\right) \text {. }
$$

(3) Define $x^{m(k)+1}=x^{m(k)}+q^{p}$, where $q^{i}$ for $i=1, \ldots, p$ is defined by

$$
q^{i}=q^{i-1}+\widetilde{B}_{k-1}^{s}\left[\left(g-\tilde{A}_{k} x^{m(k)}\right)-\widetilde{A}_{k-1} q^{i-1}\right] .
$$

(4) Set $\widetilde{B}_{k}^{s} g=x^{2 m(k)+1}$, where $x^{l}$ is defined for $l=m(k)+2, \ldots, 2 m(k)+1$ by (8.3).

It is not difficult to check that $B_{j}^{s} A_{j}=\widetilde{B}_{j}^{s} \widetilde{A}_{j}$. In addition, $\widetilde{B}_{j}^{s} F=B_{j}^{s} f$ as long as

$$
(f, \theta)=F(\theta) \text { for all } \theta \in M_{j} \text {. }
$$

This means that the multigrid algorithm using $\widetilde{B}_{j}^{s}$ as a preconditioner for (8.1) leads to the same set of iterates as the multigrid algorithm using $B_{j}^{s}$ as a preconditioner for (2.1). Thus, the above algorithm is an implementation of Algorithm $S$ provided that we use a fixed inner product $(\cdot, \cdot)$ and $R_{k}$ has the form of (8.2). Note that the use of $P_{k-1}^{0}$ in Step 3 of Algorithm $S$ has been avoided in the above implementation. This is because the natural imbedding of $M_{k}^{\prime}$ into $M_{k-1}^{\prime}$ is used. In addition, the inner products do not appear in the implementation.

Remark 8.1. The above implementation is somewhat simpler than a direct implementation of Algorithm S. This is because the prolongation and restriction parts of the above implementation only involve the relations between finite element spaces. The coefficients of the operator only affect the computation of $\widetilde{R}_{k}($ see $(5.2)$ and $(6.8))$ and $\widetilde{A}_{k}$.

\section{BIBLIOGRAPHY}

1. O. Axelsson and P. S. Vassilevski, Algebraic multilevel preconditioning methods, II, (preprint).

2. D. Bai and A. Brandt, Local mesh refinement multilevel techniques, SIAM J. Sci. Statist. Comput. 8 (1987), 109-134.

3. R. E. Bank, and C. C. Douglas, Sharp estimates for multigrid rates of convergence with general smoothing and acceleration, SIAM J. Numer. Anal. 22 (1985), 617-633.

4. R. E. Bank and T. Dupont, An optimal order process for solving finite element equations, Math. Comp. 36 (1981), 35-51.

5. D. Braess and W. Hackbusch, A new convergence proof for the multigrid method including the $V$-cycle, SIAM J. Numer. Anal. 20 (1983), 967-975. 
6. J. H. Bramble and J. E. Pasciak, New convergence estimates for multigrid algorithms, Math. Comp. 49 (1987), 311-329.

7. J. H. Bramble, J. E. Pasciak, J. Wang, and J. Xu, Convergence estimates for product iterative methods with applications to domain decomposition and multigrid, (preprint).

8. J. H. Bramble, J. E. Pasciak, and J. Xu, The analysis of multigrid algorithms for nonsymmetric and indefinite elliptic problems, Math. Comp. 51 (1988), 389-414.

9. J. H. Bramble, J. E. Pasciak, and J. Xu, Parallel multilevel preconditioners, Math. Comp. 55 (1990), 1-22.

10. J. H. Bramble, J. E. Pasciak, and J. Xu, The analysis of multigrid algorithms with nonnested spaces or noninherited quadratic forms, Math. Comp. 56 (1991), 1-34.

11. J. H. Bramble and J. Xu, Some estimates for a weighted $L^{2}$ projection, Math. Comp. 56 (1991), 463-476.

12. A. Brandt, Algebraic multigrid theory: the symmetric case, Appl. Math. Comput. 19 (1986), 23-56.

13. A. Brandt, Multi-level adaptive solutions to boundary-value problems, Math. Comp. 31 (1977), 333-390.

14. W. Hackbusch, Multi-grid methods and applications, Springer-Verlag, New York, 1985.

15. R. B. Kellogg, Interpolation between subspaces of a Hilbert space, Tech. Note BN-719, Univ. of Maryland, Inst. Fluid Dynamics and Appl. Math., 1971.

16. M. Kočvara and J. Mandel, A multigrid method for three-dimensional elasticity and algebraic convergence estimates, Appl. Math. Comput. 23 (1987), 121-135.

17. J. F. Maitre and F. Musy, Algebraic formalization of the multigrid method in the symmetric and positive definite case- a convergence estimation for the V-cycle, Multigrid Methods for Integral and Differential Equations (D. J. Paddon and H. Holstein, eds.), Clarendon Press, Oxford, 1985.

18. J. Mandel, Étude algébrique d'une méthode multigrille pour quelques problèmes de frontière libre, C.R. Acad. Sci. Paris Sér. I. Math. 298 (1984), 469-472.

19. J. Mandel, Multigrid convergence for nonsymmetric, indefinite variational problems and one smoothing step, (Proc. Copper Mtn. Conf. Multigrid Methods), Applied Math. Comput. 19 (1986), 201-216.

20. J. Mandel, S. F. McCormick, and R. Bank, Variational multigrid theory, Multigrid Methods (S. McCormick, ed.), SIAM, Philadelphia, PA, 1987, pp. 131-178.

21. P. Vassilevski, Iterative methods for solving finite element equations based on multilevel splitting of the matrix, Bulgarian Academy of Sciences, Sofia, Bulgaria, (preprint).

22. J. Xu, Convergence estimates for some multigrid algorithms, Proc. 1989 Houston Domain Decomp. Methods Conf. Third Internat. Sympos. Domain Decomposition Methods for Partial Differential Equations (T. Chan, R. Glowinski, J. Periaux, and O. Widlund, eds.), SIAM, Philadelphia, PA, 1990, pp. 174-187.

23. J. Xu, Theory of multilevel methods, Ph.D. Thesis, Cornell University and Penn State University, Dept. Math. Rep AM-48, 1989.

Department of Mathematics, Cornell University, Ithaca, New York 14853

E-mail address: bramble@mathvax.msi.cornell.edu

Department of Applied Science, Brookhaven National Laboratory, Upton, New York 11973

E-mail address: pasciak@bnl.gov

Mathematics Department, The University of Wyoming, Laramie, Wyoming 82071

Department of Mathematics, Pennsylvania State University, University Park, PennSYLVANIA 16802 